A comparison of water vapor quantities from model short-range forecasts and ARM observations

J. J. Hnilo

March 22, 2006 
This document was prepared as an account of work sponsored by an agency of the United States Government. Neither the United States Government nor the University of California nor any of their employees, makes any warranty, express or implied, or assumes any legal liability or responsibility for the accuracy, completeness, or usefulness of any information, apparatus, product, or process disclosed, or represents that its use would not infringe privately owned rights. Reference herein to any specific commercial product, process, or service by trade name, trademark, manufacturer, or otherwise, does not necessarily constitute or imply its endorsement, recommendation, or favoring by the United States Government or the University of California. The views and opinions of authors expressed herein do not necessarily state or reflect those of the United States Government or the University of California, and shall not be used for advertising or product endorsement purposes.

This work was performed under the auspices of the U.S. Department of Energy by University of California, Lawrence Livermore National Laboratory under Contract W-7405-Eng-48. 


\section{A comparison of water vapor quantities from model short-range forecasts and ARM observations.}

March 17, 2006

J. Hnilo and the CAPT group Lawrence Livermore National Laboratory

Livermore, California

Work supported by the U.S. Department of Energy,

Office of Science, Office of Biological and Environmental Research 


\section{CONTENTS}

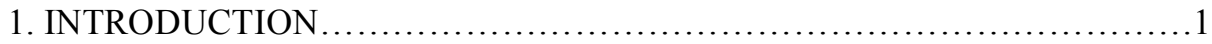

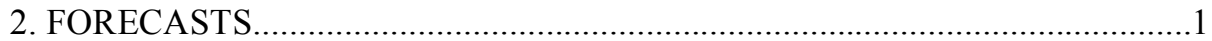

a. MODEL DATA........................................................................ 1

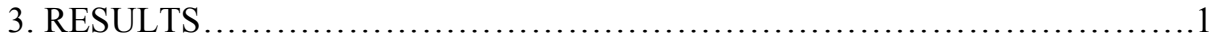

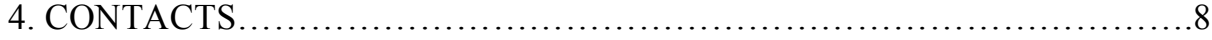

\section{FIGURES}

1. Time series of column integrated water vapor $(\mathrm{kg} \mathrm{m}-2)$, for two time periods at the three ARM sites, DAR (top), NSA (middle) and SGP (bottom). In each panel black is the CAM3, red is the AM2 model and blue are the merged-sounding data. For the top panel an additional dotted blue line is shown and this is the NASADAO integrated water.

2. The time evolution of specific humidity (kg kg-1) for OBS (top), AM2 (middle) and CAM3 (bottom) at the NSA .........................................

3. Mean Bias (OBS - Model) at Dar (top), NSA (middle) and SGP (bottom) in $\mathrm{gm} / \mathrm{kg}-1, \mathrm{CAM} 3$ = black, AM2= red. Note horizontal scales differ between

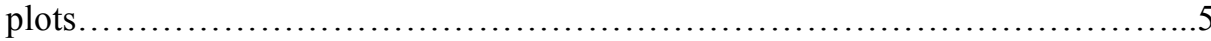

4. Correlation Coefficients for specific humidity between OBS and Model for the 37 pressure levels DAR (top, $\mathrm{N}=177$ ), NSA (middle, $\mathrm{N}=177$ ) and SGP (bottom, $\mathrm{N}=233$ ), CAM3 =black,AM2=red

5. The Root Mean Square error of specific humidity for the 37 pressure levels in gm kg-1 at DAR (top), NSA (middle) and SGP (bottom), $\mathrm{CAM} 3=$ black, $\mathrm{AM} 2=$ red 


\section{INTRODUCTION:}

Model evolution and improvement is complicated by the lack of high quality observational data. To address a major limitation of these measurements the Atmospheric Radiation Measurement (ARM) program was formed. For the second quarter ARM metric we will make use of new water vapor data that has become available, and called the "Mergedsounding" value added product (referred to as OBS, within the text) at three sites: the North Slope of Alaska (NSA), Darwin Australia (DAR) and the Southern Great Plains (SGP) and compare these observations to model forecast data. Two time periods will be analyzed March 2000 for the SGP and October 2004 for both DAR and NSA. The merged-sounding data have been interpolated to 37 pressure levels (e.g., from $1000 \mathrm{hPa}$ to $100 \mathrm{hPa}$ at $25 \mathrm{hPa}$ increments) and time averaged to 3hourly data for direct comparison to our model output.

\section{FORECASTS:}

The Climate Change Prediction Programs (CCPP) and ARM Parameterization Testbed (CAPT) is a joint project of the U.S. Department of Energy's Office of Science/Biological and Energy Research (BER). We use analyses of global weather from numerical weather prediction (NWP) centers, in conjunction with field observations such as those provided by ARM, to evaluate parameterizations of sub-gridscale processes in global climate models. Simply stated, we run realistically initialized climate models in forecast mode to determine their initial drift from the NWP analyses and/or from the available field data, thereby gaining insights on model parameterization deficiencies.

The CAPT protocol, which is analagous to a common NWP approach for development of forecast models, is also potentially useful for diagnosing parameterization problems that may produce systematic model errors on climate time scales. The goal is to adapt this NWPinspired technique to a degree sufficient for its practical application in the development cycles of climate models.

\section{a. MODEL DATA:}

Forecasts are initialized at 00UTC daily and run for approximately three days in length. After an initial time period of 12 hours, where the model comes to equilibrium with its new imposed conditions we sample these forecasts for the next 24 hours (e.g., hours 12-36 of the forecast), we the generate an appended time series of the model output and compare these results to robust high frequency (e.g., ARM data). For our two time periods of interest, we use differing initializing data. For march 2000 (SGP) we use the European Centre for Medium Range Weather Forecasts ERA40 reanalyses data and for the October 2004 (NSA and DAR) we use the National Aeronautics and Space Administration-Data Assimilation Office (NASA-DAO) analyses. Within this work we compare results from the National Center for Atmospheric Research, CAM3.1 model and the Geophysical Fluid Dynamics Laboratory AM2 model. Model output is a distance weighted value from the closest four model grid points to the ARM station locations.

\section{RESULTS:}

Integrated Atmospheric water vapor content are shown in figure 1 for the three ARM sites. For the Darwin site (Figure 1, top panel) we find the Bias (as OBS-Model) and Root Mean Square (RMS) to be 5.3 and $7.53 \mathrm{~kg} \mathrm{~m}-2$ for the AM2 and 8.7 and $10.1 \mathrm{~kg} \mathrm{~m}-2$ for the CAM3. The correlation between the merged sounding data and model output are $r=0.44$ and $\mathrm{r}=0.50$ for the AM2 and CAM3 respectively. These differences at Darwin are large. Perhaps some of the explanation can be seen from our inclusion of the NASA-DAO integrated water 
content. It can be noted -that the models seem to agree better with the initialization analyses than the merged-sounding data, leading us to believe there may be problems with the tropical moisture within this analysis. For the NSA we find the Bias and RMS to be -0.65 and $1.69 \mathrm{~kg}$ $\mathrm{m}-2$ for the AM2 and -0.09 and $1.47 \mathrm{~kg} \mathrm{~m}-2$ for the CAM3. The correlation values are $\mathrm{r}=0.78$ and $\mathrm{r}=0.82$ for AM2 and CAM3 respectively. For the SGP we find bias and RMS values of -0.20 and $2.81 \mathrm{~kg} \mathrm{~m}-2$ for AM2 and 0.67 and $3.14 \mathrm{~kg} \mathrm{~m}-2$ for CAM3. The correlation values are $\mathrm{r}=0.90$ and $\mathrm{r}=0.86$ for AM2 and CAM3 respectively.

Figure two is a three panel plot showing the time evolution of specific humidity (kg kg-1) at the NSA. The top plot is the merged sounding data and the middle is AM2 and the bottom is the CAM3. This figure is included only for NSA and is solely to show that all subsequent line plots (for this and the other sites) are derived from such two dimensional fields.

The Bias (e.g., OBS - Model) at the three sites for the two differing time periods is shown in figure three (for ease of understanding they have been converted to gm kg-1). The top panel is DAR where both models clearly show a dry Bias extending up to approximately $700 \mathrm{hPa}$ with the CAM3 being to dry in excess of $3.0 \mathrm{gm} \mathrm{kg}-1$ and the AM2 in excess of $2.0 \mathrm{gm} \mathrm{kg}-1$. The middle and lower panel exhibit Biases much smaller than those shown in the first panel for Darwin. The middle panel shows NSA where both models exhibit too much specific humidity the AM2 by $0.2 \mathrm{gm} \mathrm{kg}-1$ and CAM3 by $0.24 \mathrm{gm} \mathrm{kg}-1$ at approximately $800 \mathrm{hPa}$ with varying degrees of successful simulation of specific humidity as one nears the surface. The bottom panel for the SGP shows the AM2 model being to dry near the surface by $0.2 \mathrm{gm} \mathrm{kg}-1$ and then slightly too moist $850 \mathrm{hPa}$ by $0.4 \mathrm{gm} \mathrm{kg}-1$. For the CAM3 it is clearly to dry near $800 \mathrm{hPa}$ by approximately $0.3 \mathrm{gm} \mathrm{kg}-1$ and then to moist at $500 \mathrm{hPa}$ by $0.25 \mathrm{gm} \mathrm{kg}-1$.

The correlation between the OBS and model output can be seen in figure four. For DAR the correlative values are lower near the surface and largest near $300 \mathrm{hPa}$, which differs from the values at both SGP and NSA where the correlations are largest near the surface. For both NSA and SGP the maximum value appears near the surface with a general trend of decrease in correlation with height. The Root Mean Square Error can be seen in figure five. For all cases there is a general maximum RMS value near $800 \mathrm{hPa}$ with decreasing values as one goes higher in the atmosphere and closer to the surface.

We have successfully compared the model forecast data to the new ARM "merged-sounding" data, which fulfills the second quarter metric. Results show that there is good agreement in these measures for the SGP and NSA and significantly lesser agreement for the DAR site. 

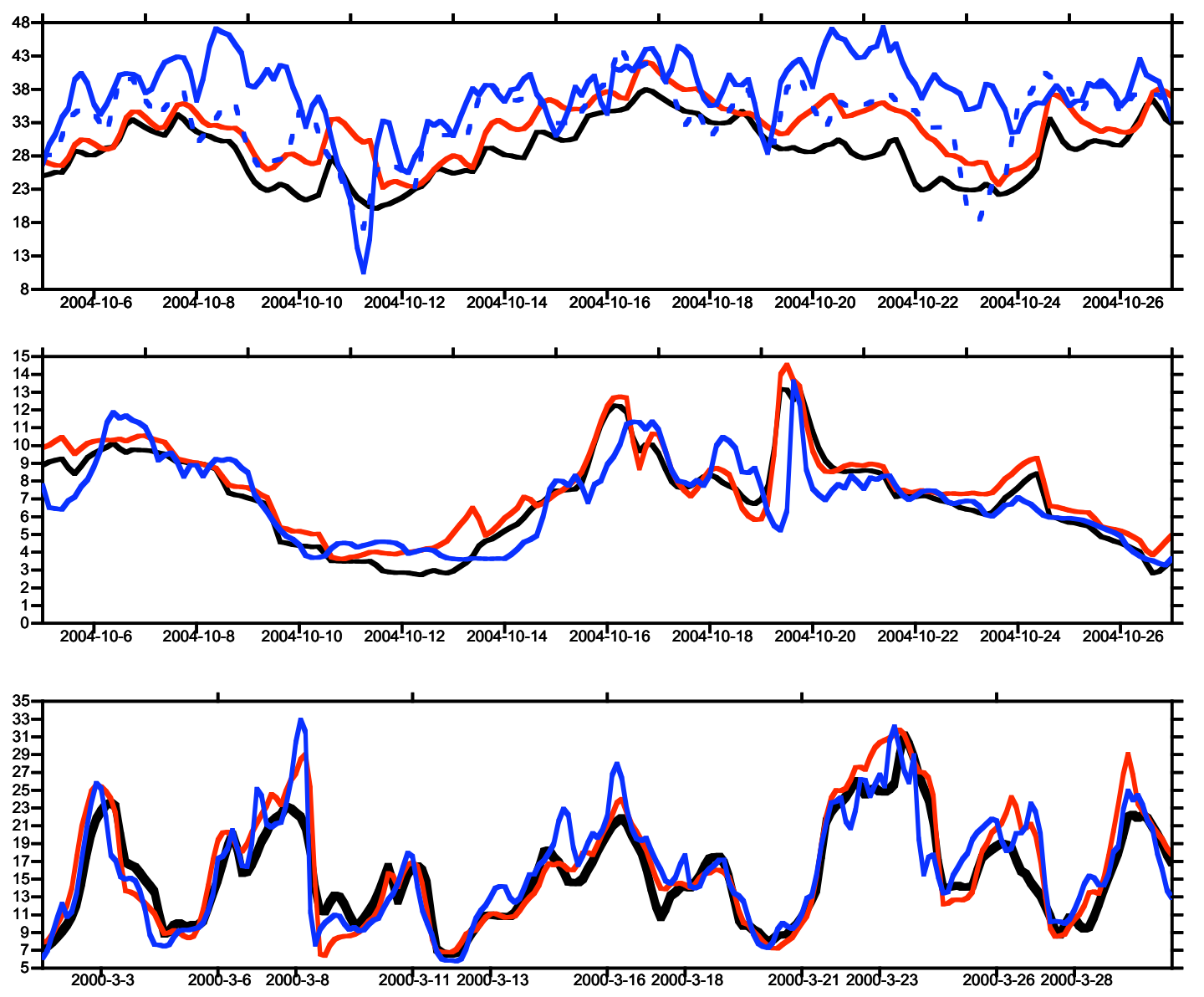

Figure 1. Time series of column integrated water vapor $(\mathrm{kg} \mathrm{m}-2)$, for two time periods at the three ARM sites, DAR (top), NSA (middle) and SGP (bottom). In each panel black is the CAM3, red is the AM2 model and blue are the merged-sounding data. For the top panel an additional dotted blue line is shown and this is the NASA-DAO integrated water 

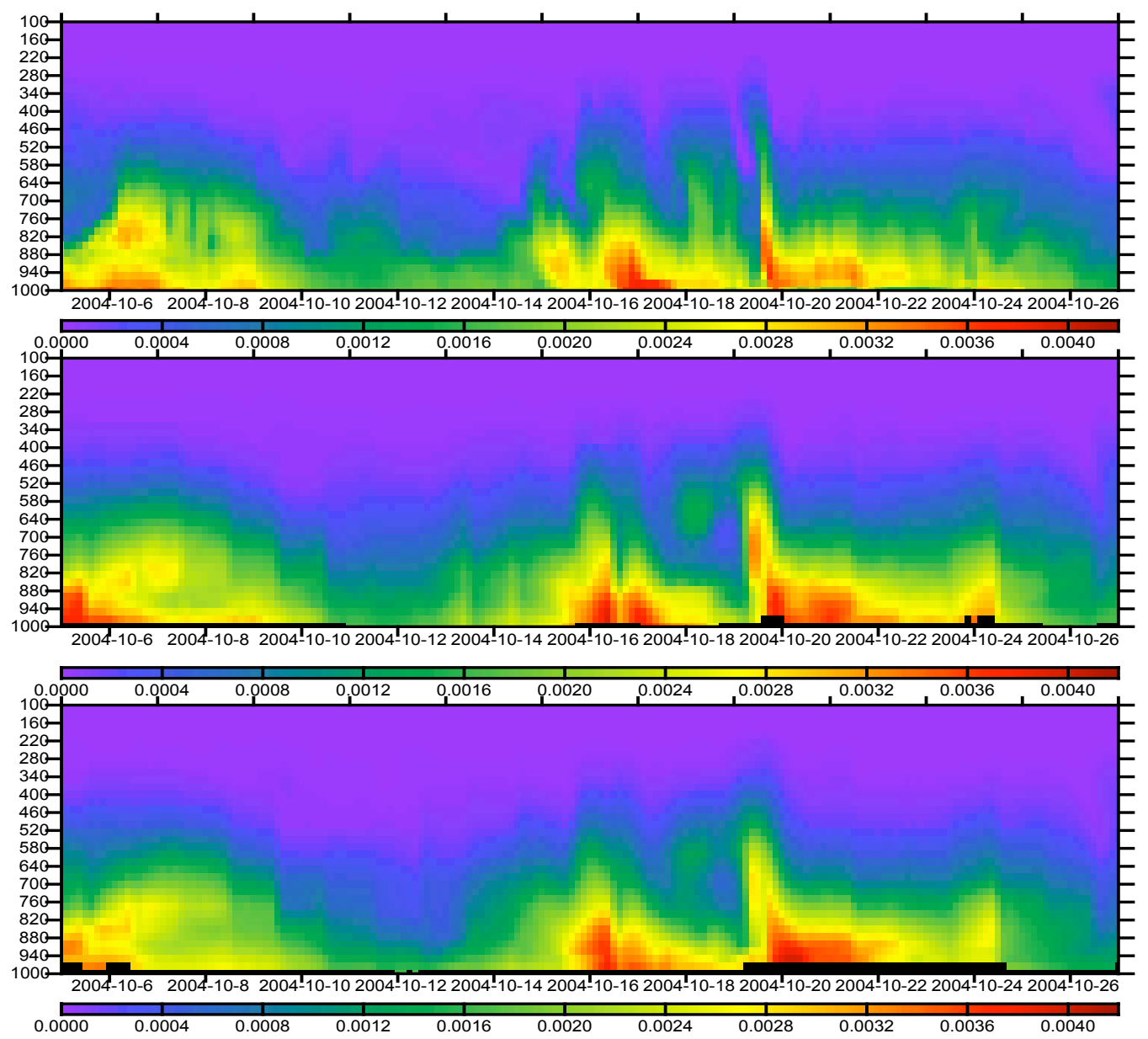

Figure 2. The time evolution of specific humidity (kg kg-1) for OBS (top), AM2 (middle) and CAM3 (bottom) at the NSA. 

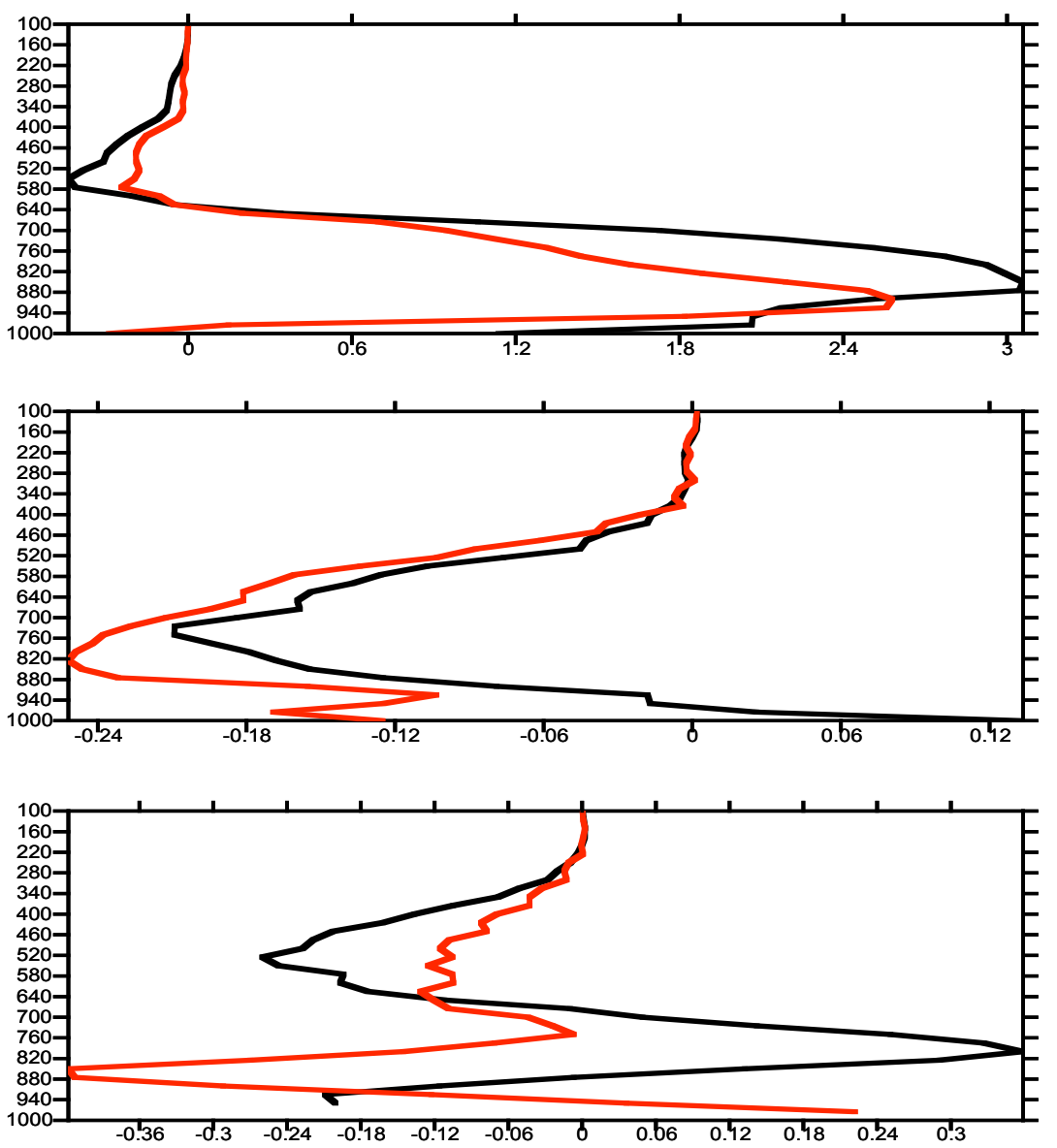

Figure 3. Mean Bias (OBS - Model) at Dar (top), NSA (middle) and SGP (bottom) in $\mathrm{gm} / \mathrm{kg}-1, \mathrm{CAM} 3=$ black, $\mathrm{AM} 2=$ red. Note horizontal scales differ between pictures. 

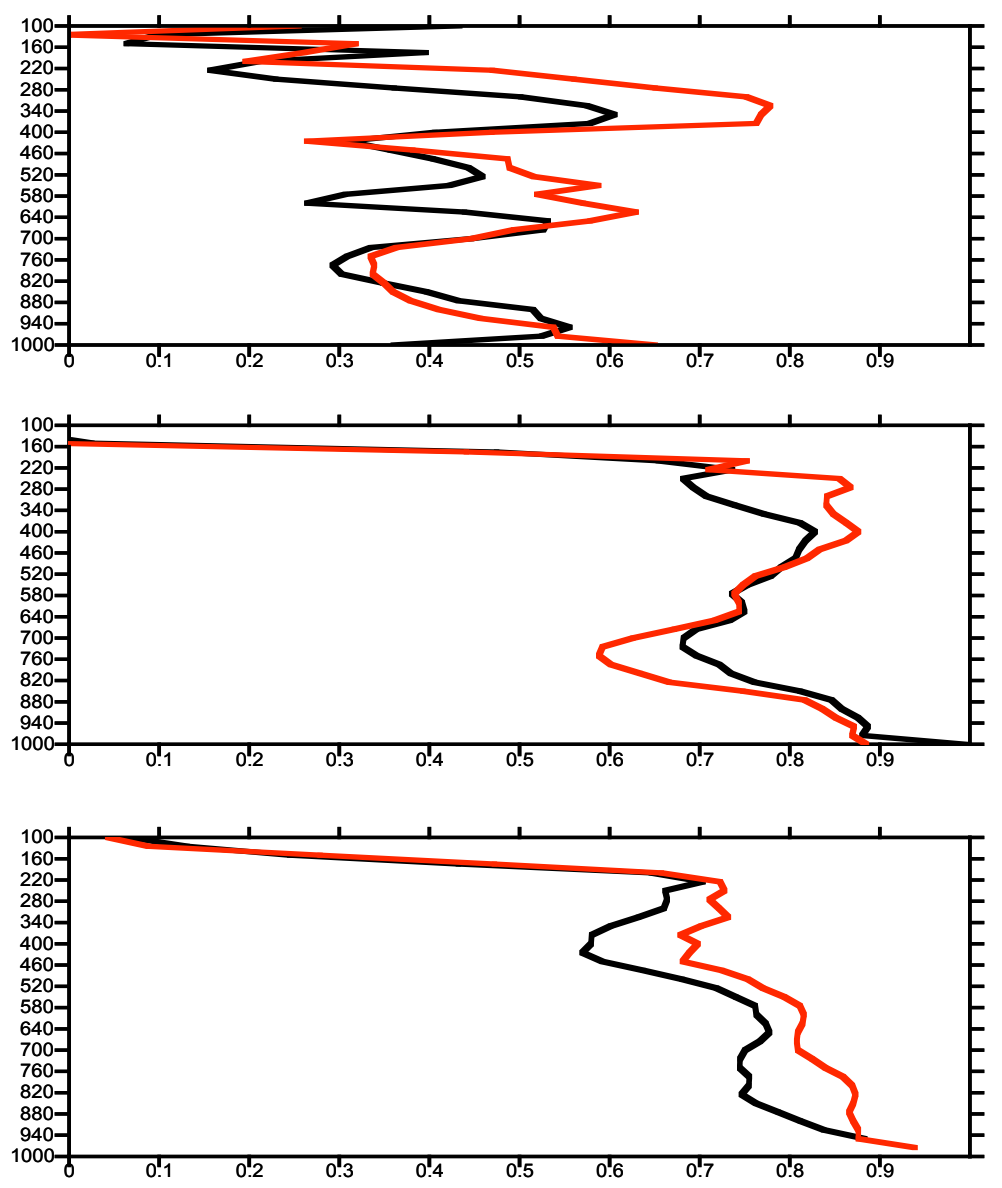

Figure 4. Correlation Coefficients for specific humidity between OBS and Model for the 37 pressure levels DAR (top, $\mathrm{N}=177$ ), NSA (middle, $\mathrm{N}=177$ ) and SGP (bottom, $\mathrm{N}=233$ ), $\mathrm{CAM} 3$ = black, AM2= red. 

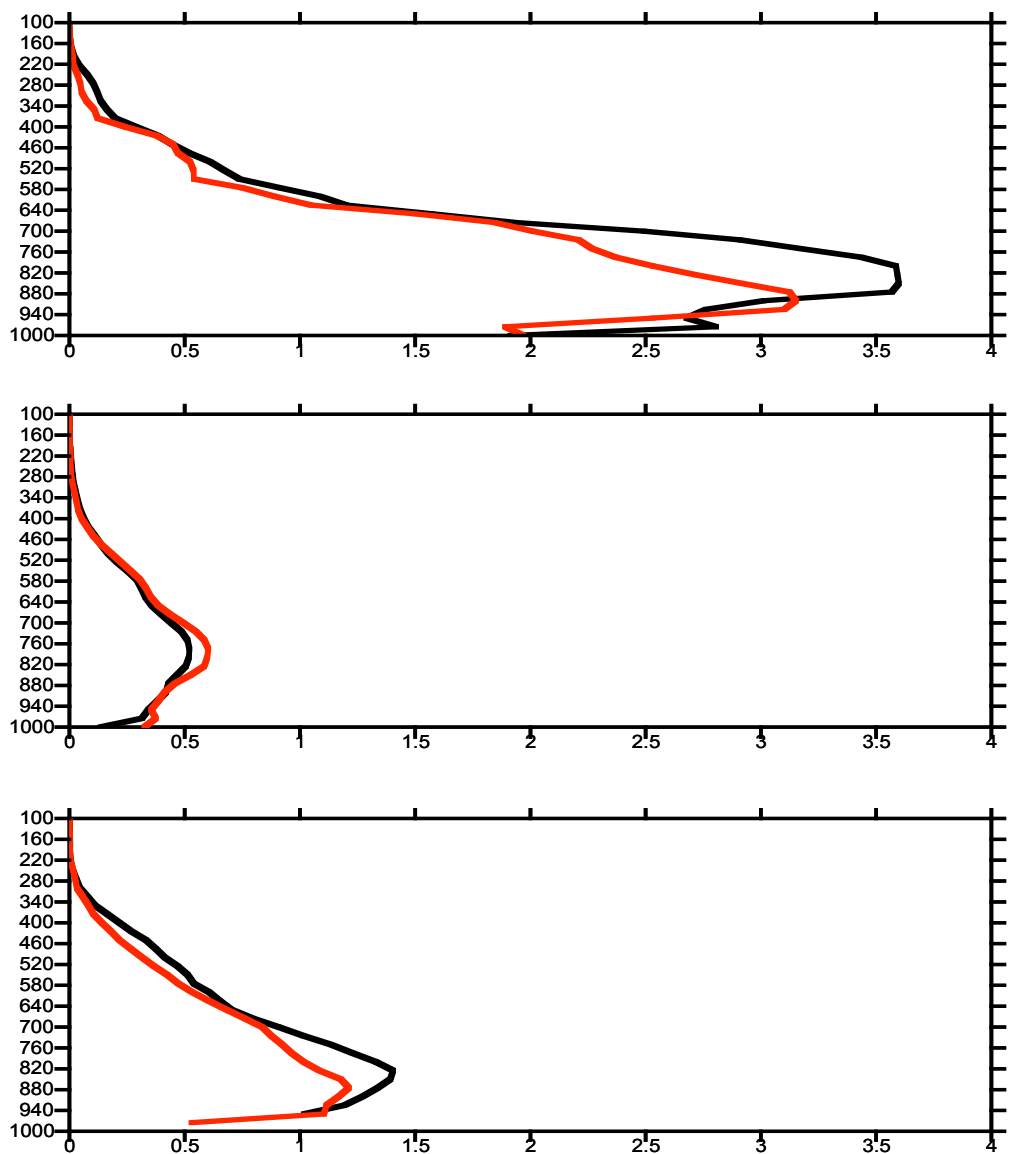

Figure 5. The Root Mean Square error of specific humidity for the 37 pressure levels in gm kg-1 at DAR (top), NSA (middle) and SGP (bottom), $\mathrm{CAM} 3=$ black, $\mathrm{AM} 2=$ red. 
4. CONTACTS

J. Hnilo, hnilo1@Ilnl.gov, （925)-423-2268

S. Klein, klein21@Ilnl.gov, (925)-423-9777 